\title{
MGBPv2: Scaling Up Multi-Grid Back-Projection Networks
}

\author{
Pablo Navarrete Michelini, Wenbin Chen, Hanwen Liu, Dan Zhu \\ BOE Technology Co., Ltd. \\ \{pnavarre, chenwb, liuhanwen, zhudan\}@boe.com.cn
}

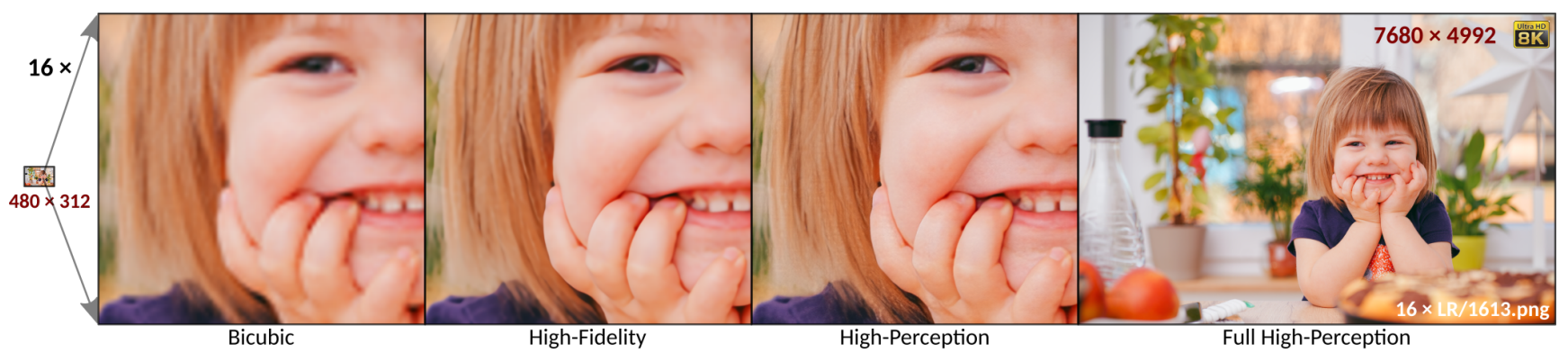

Figure 1: Large upscaling factors are needed to convert standard-definition (SD) to ultra-high-definition (UHD) resolutions. In the task of Extreme Super-Resolution (zooming 16×), our proposed MGBPv2 system can achieve a good balance between high fidelity (images close to the original content) and high perceptual quality (images that look real through artificial details).

\section{Abstract}

Here, we describe our solution ${ }^{1}$ for the AIM-2019 Extreme Super-Resolution Challenge, where we won the $1^{\text {st }}$ place in terms of perceptual quality (MOS) similar to the ground truth and achieved the $5^{\text {th }}$ place in terms of highfidelity (PSNR). To tackle this challenge, we introduce the second generation of MultiGrid BackProjection networks (MGBPv2) whose major modifications make the system scalable and more general than its predecessor. It combines the scalability of the multigrid algorithm and the performance of iterative backprojections. In its original form, $M G B P$ is limited to a small number of parameters due to a strongly recursive structure. In $M G B P v 2$, we make full use of the multigrid recursion from the beginning of the network; we allow different parameters in every module of the network; we simplify the main modules; and finally, we allow adjustments of the number of network features based on the scale of operation. For inference tasks, we introduce an overlapping patch approach to further allow processing of very large images (e.g. $8 \mathrm{~K}$ ). Our training strategies make use of a multiscale loss, combining distortion and/or perception losses on the output as well as downscaled output images. The final system can balance between high quality and high performance.

\section{Introduction}

Image upscaling has been studied for decades and remains an active topic of research because of constant

\footnotetext{
${ }^{1}$ Code available at https://github.com/pnavarre/mgbpv2
}

technological advances in digital imaging. One scenario where image upscaling is now more demanding arises in digital display technologies, where new standards like BT.2020 [18] are introduced. The resolution of digital displays has experienced a tremendous growth over the past few decades, as shown in Figure 2. The transition between different formats leads to a challenging problem. On one hand, large amount of digital content still exist in popular old standards such as standard-definition (SD). On the other hand, the latest display technologies (e.g. $4 \mathrm{~K}, 8 \mathrm{~K}$ and above) are expected to show this content with reasonable quality. Standard upscaling technologies are clearly insufficient for this purpose. While a $2 \times$ upscaler maps 1 input pixel into 4 output pixels, a $16 \times$ upscaler maps 1 input pixel into 256 output pixels, which already contain small images. The problem demands advanced solutions that are capable of understanding the image content and filling in these large pieces of images with visually appealing and consistent information. In particular, large upscaling factors are needed to convert SD to ultra high-definition (UHD) resolutions. For example, to upscale from a popular width of 640 pixels (VGA) to $8,192(8 \mathrm{~K})$ we need a factor greater than $12 \times$. And from 640 pixels to $10,240(10 \mathrm{~K})$, which is used by some of the most advanced modern displays [4,3], we need a $16 \times$ factor. Thus, extreme upscaling represents a real problem in current market and it is expected to persist and become even more challenging with the rapid development of new technologies.

The challenge on Extreme Super-Resolution proposed at the 2019 ICCV workshop on Advances in Image Manipulation (AIM-2019) [17] was the first of its kind to tackle this problem. That is, the task of increasing the resolution of an 


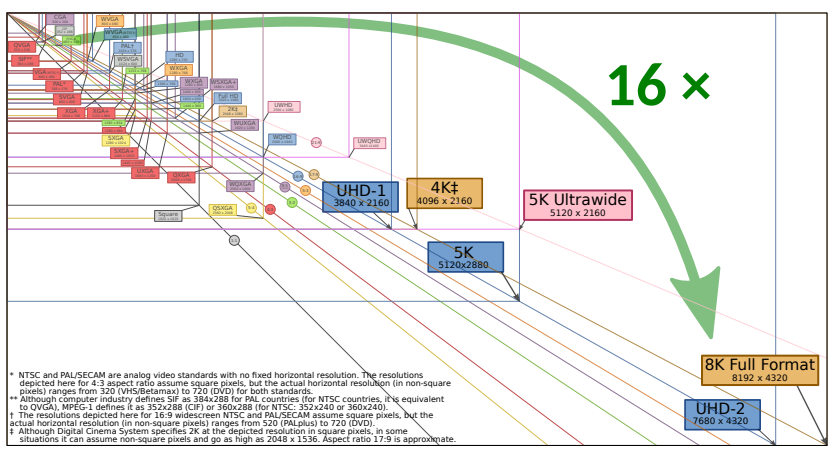

Figure 2: Extreme Super-Resolution is a technology necessary to connect the extremes in the dramatic growth of standard resolutions. Source: Wikimedia CC BY-SA.

input image with a magnification factor $16 \times$ based on a set of prior examples of low and corresponding high resolution images. The challenge provided a novel DIVerse up to $8 \mathrm{~K}$ resolution images dataset (DIV8K) with a large diversity of content. Two targets were proposed:

- Track 1: Fidelity, the aim is to obtain a solution capable to produce high resolution results with the best fidelity (PSNR) to the ground truth.

- Track 2: Perceptual, the aim is to obtain a solution capable to produce high resolution results with the best perceptual quality similar to the ground truth.

These two targets are motivated by the so-called perception-distortion trade-off established by Blau and Michaeli in [2]. That is, both targets cannot be achieved at the same time, one must compromise perceptual quality to improve fidelity and vice versa. The trade-off motivated the Perceptual Image Restoration and Manipulation (PIRM) workshop at ECCV-2018 [1] that strongly confirmed and gave further insight into this fundamental principle.

In the Extreme-SR competitions, we identified two major challenges: performance scalability, due to the large size of the output images; and content scalability, due to the diverse scale of image content. Our solution was built upon the MultiGrid BackProjection (MGBP) network proposed in $[16,15]$, combining both the iterative backprojection and multigrid algorithms. First, the iterative backprojection algorithm provides performance. With solid ground in the classical theory of image super-resolution (SR), its deep-learning versions have succeeded in competitions. Examples include DBPN [5] (which won the NTIRE-2018 challenge on $8 \times$ SR and the Region-2 track of PIRM-SR 2018[1]) and MGBP [15] (which won the $2^{\text {nd }}$ place in the Region-3 track, i.e. best perception, of PIRMSR 2018[1]). Second, the multigrid algorithm provides scalability. Here, it is worth noting that multigrid is one of the most popular algorithms in the field of PDE and numerical solvers[19], but its name is often misinterpreted in other fields as merely meaning "multilevel". Multigrid is one of the few known methods that can solve numerical systems of linear equations with optimal computational complexity (linear in the number of unknowns). The MGBP algorithm does not work as a linear solver, but borrows the multigrid recursion to scale the iterative backprojection algorithm. The recursion unfolds into a convenient structure, in which the computational complexity grows linearly with the image size. This is visible in the diagram of the system (see for example Figure 3), where the number of modules is maximum at the lowest resolution and decreases exponentially as the resolution increases.

Despite its convenient properties, the original formulation of MGBP does not scale well for extreme upscaling factors. This is due to two problems: first, the small number of parameters in MGBP limits its performance on image quality compared to large models; second, the recursive structure forces the number of network features to remain constant along scales. Our main contribution is to redesign MGBP to fix these problems. We summarize our contributions as follows:

- We propose to make full use of the multigrid recursion from the beginning of the network.

- We propose to simplify the main modules and allow different parameters for every instance in the network.

- We propose a strategy to merge overlapping patches in inference to further allow processing of very large images.

- We propose training strategies at multiple scales, combining distortion and/or perception losses on the output as well as downscaled output images.

\section{System Architecture}

Without loss of generality, we tackle the image enhancement problem with an input resolution equal to the output resolution. For the super-resolution task we input the low resolution image upscaled $16 \times$ using a bicubic method. This helps to make the system become more general for applications and simplifies the process of generating pairs of input/output patches during training.

We propose the MGBPv2 algorithm shown in Algorithm 1. In Figure 3 we display the diagram of the algorithm unfolded for $\mu=2$ and $L=5$. The Analysis and Synthesis modules covert an image into features space and vice-versa using single convolutional layers. The Upscaler and Downscaler modules are composed of single strided (transposed and conventional) convolutional layers. An important observation in Algorithm 1 that makes a significant difference with the original MGBP is the use of a tag label to differentiate each Upscaler and Downscaler module. This simple 


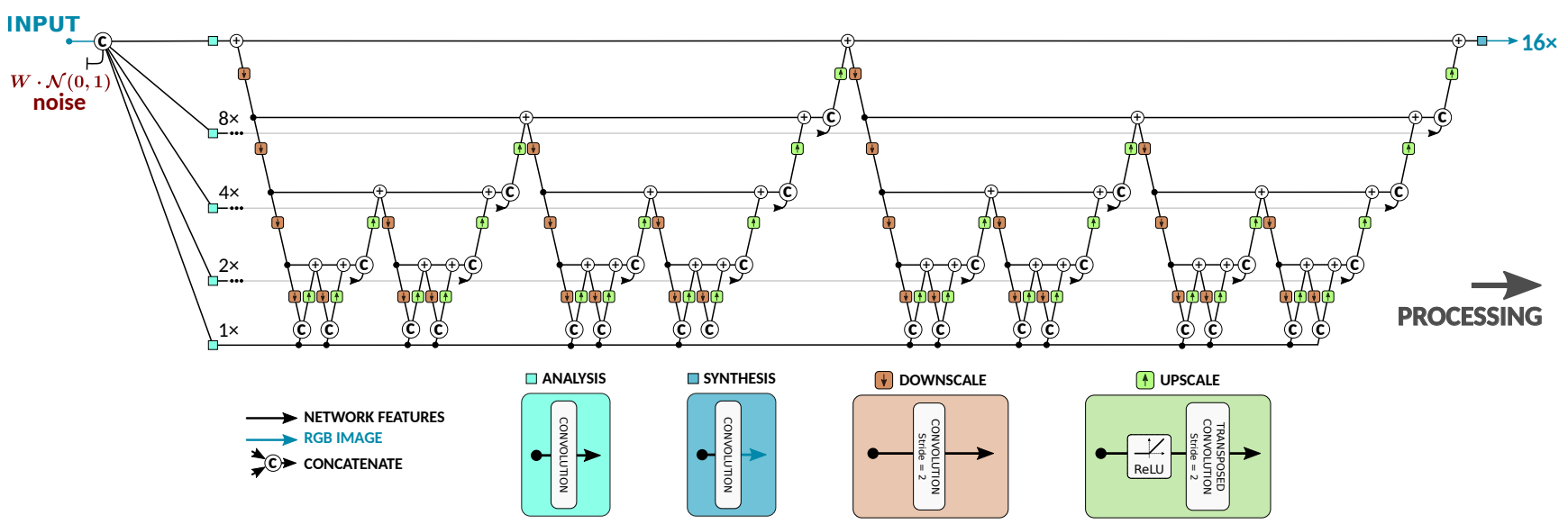

Figure 3: Diagram of the MultiGrid BackProjection version 2 (MGBPv2) network unfolded from Algorithm 1 with $\mu=2$.

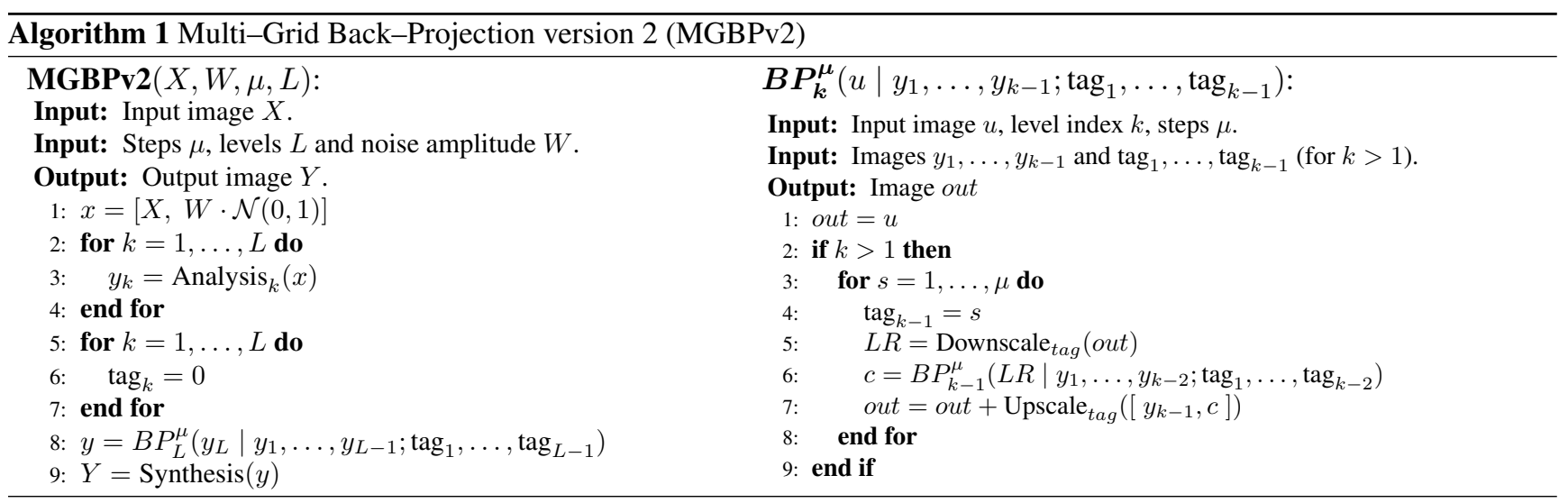

trick makes every Upscaler and Downscaler module different in terms of parameters and hyper-parameters. In particular, we now set the number of features in convolutional layers to $256,192,128,92,48$ and 9 from low to high resolution levels, allowing large images to be processed at high resolutions. The system can be initialized by a dry run of Algorithm 1 where no computation is performed and modules are defined with their correspondent tag labels.

For the Perceptual track we used a single MGBPv2 system configured with filters of $3 \times 3$. For the Fidelity track we used an ensemble solution that sums the outputs of 3 systems with identical configuration except for different filter sizes: $3 \times 3,5 \times 5$ and $7 \times 7$.

We concatenate a single noise channel, $W \cdot \mathcal{N}(0,1)$, to the bicubic upscaled input. The amplitude of the noise, $W \in \mathbb{R}$, is set to zero in the Fidelity track and its purpose is to help in the adversarial training for the Perceptual track. Following the design of the generative MGBP algorithm in [15], the noise activates and deactivates the generation of artificial details. As opposed to [15] we now generate a single noise channel at the highest resolution, that moves along with the input image to enter the network at different scales by using Analysis modules with different strides. In [15], different i.i.d. noise channels are generated at each resolution. This change becomes necessary during inference, where we merge the output of several overlapping patches and therefore we need to use noise images that overlap in the same way as the input image.

For the discriminator in adversarial training we use the system shown in Figure 4. Here, we use 4-layer sequential CNNs with $3 \times 3$ filters and stride 1 except for the last layer that uses stride 2 to downscale the features. The system resembles the design of the discriminator in [15] with two major differences: first, the system in Figure 4 is not recursive (all CNNs have different parameters); and second, the system uses a single high-resolution input that is downsized using fixed bicubic downscalers to enter at different levels of the system. We use the same variance normalization and shift correlator $(\mathrm{VN}+\mathrm{SC})$ module from [15].

\section{Training Strategies}

We denote $Y_{W=0}$ and $Y_{W=1}$ the outputs of the generator architecture using noise amplitudes $W=0$ and $W=1$, respectively. Let $S_{f}$ represent a bicubic downscaler that re- 

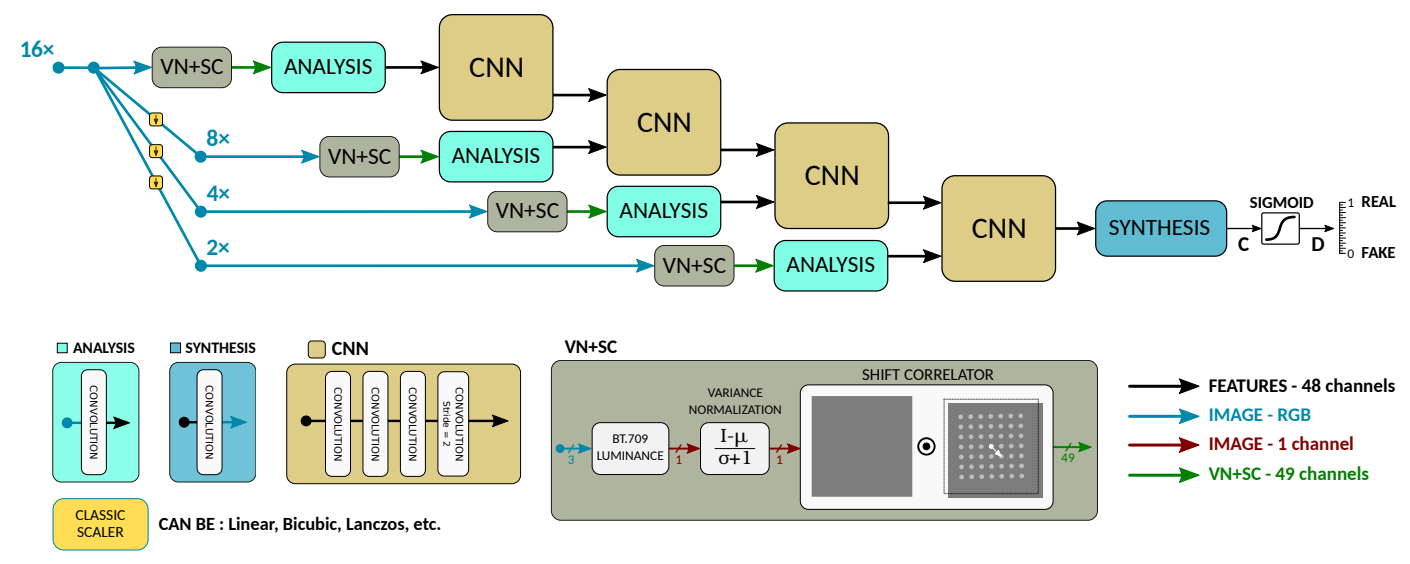

Figure 4: Discriminator system used for adversarial training. The high resolution input image is downsized with standard bicubic downscalers to enter the system at different scales. CNN modules do not share parameters.

duces the resolution by a factor $f$. We know that the original image was downscaled with $S_{16}$. Then, our training strategies in terms of loss functions are defined follows:

Fidelity Track: We use $\mathcal{L}^{L 1}(x, y)=\mathbb{E}[|x-y|]$ at several resolutions to define the total loss function:

$$
\begin{aligned}
\mathcal{L}(Y, X ; \theta)= & \mathcal{L}^{L 1}\left(Y_{W=0}, X\right)+ \\
& \mathcal{L}^{L 1}\left(S_{2}\left(Y_{W=0}\right), S_{2}(X)\right)+ \\
& \mathcal{L}^{L 1}\left(S_{4}\left(Y_{W=0}\right), S_{4}(X)\right)+ \\
& \mathcal{L}^{L 1}\left(S_{8}\left(Y_{W=0}\right), S_{8}(X)\right)+ \\
& \mathcal{L}^{L 1}\left(S_{16}\left(Y_{W=0}\right), S_{16}(X)\right) .
\end{aligned}
$$

After every epoch we evaluate the current model using $\mathcal{L}^{L 2}(x, y)=\mathbb{E}\left[(x-y)^{2}\right]$ in the validation metric:

$$
\mathcal{V}(Y ; \theta)=\mathcal{L}^{L 2}\left(Y_{W=0}, X\right) .
$$

We recorded the best models according to this metric (directly related to PSNR) during the training process and we performed further manual tests on larger images afterwards.

Perceptual Track: We follow the design of multi-scale loss from the generative MGBP in [15] with some simplifications to improve training performance. One significant change is in the adversarial loss. We changed the standard GAN used in [15] to a Relativistic GAN [7] following the recommendation in [20]. Our total loss is given by:

$$
\begin{aligned}
\mathcal{L}(Y, X ; \theta)= & 0.001 \cdot \mathcal{L}_{G}^{R S G A N}\left(Y_{W=1}\right)+ \\
& 10 \cdot \mathcal{L}^{L 1}\left(S_{16}\left(Y_{W=1}\right), S_{16}(X)\right)+ \\
& 0.1 \cdot \mathcal{L}^{C X}\left(Y_{W=1}, X\right)+ \\
& 10 \cdot \mathcal{L}^{L 1}\left(Y_{W=0}, X\right)+ \\
& 10 \cdot \mathcal{L}^{L 1}\left(S_{16}\left(Y_{W=0}\right), S_{16}(X)\right) .
\end{aligned}
$$

Here, $\mathcal{L}^{C X}$ is the contextual loss as defined in [12] using features from conv3-4 of a VGG-19 network as suggested in [11]. Ablation tests in [15] have shown the effectiveness of this loss function to improve perceptual quality while maintaining a reasonable level of distortion. Next, the Relativistic GAN loss follows the definition in [7], given by:

$$
\begin{aligned}
\mathcal{L}_{D}^{R S G A N} & =-\mathbb{E}_{(R, F)}[\log (\operatorname{sigmoid}(C(R)-C(F)))], \\
\mathcal{L}_{G}^{R S G A N} & =-\mathbb{E}_{(R, F)}[\log (\operatorname{sigmoid}(C(F)-C(R)))] .
\end{aligned}
$$

Here, $C$ is the output of the discriminator before the sigmoid function, as shown in Figure 4. And $R$ and $F$ are the sets of real and fake inputs to the discriminator, given by:

$$
\begin{aligned}
& F=\left\{Y_{W=1}, S_{2}\left(Y_{W=1}\right), S_{4}\left(Y_{W=1}\right), S_{8}\left(Y_{W=1}\right)\right\}, \\
& R=\left\{X, S_{2}(X), S_{4}(X), S_{8}(X)\right\} .
\end{aligned}
$$

After every epoch we evaluated the current model according to the validation metric based on the NIQE[13] index:

$$
\begin{gathered}
\mathcal{V}(Y ; \theta)=\mathbb{E}\left[\operatorname{NIQE}\left(Y_{W=1}\right)+\operatorname{NIQE}\left(S_{2}\left(Y_{W=1}\right)\right)+\right. \\
\left.\operatorname{NIQE}\left(S_{4}\left(Y_{W=1}\right)\right)\right] .
\end{gathered}
$$

This metric works as a simple rule to help identify models that generate realistic images in the full resolution, as well as two levels of resolutions below. In other words, we want output images to look real as seen in a display device both from close and far away distances. We performed further subjective tests on larger images to select the best models.

\section{Inference Strategies}

To upscale large images we propose a patch based approach in which we average the output of overlapping patches taken from the bicubic upscaled input. First, we divide input images into overlapping patches (of same size as training patches) as shown in Figure 6; second, we multiply each output by a weight decreasing as the distance to 


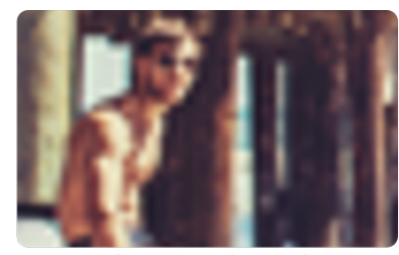

Bicubic / 10.85/18.27

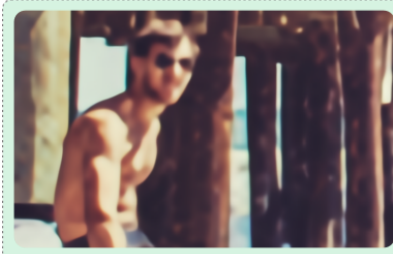

Fidelity / 8.43 / 10.29

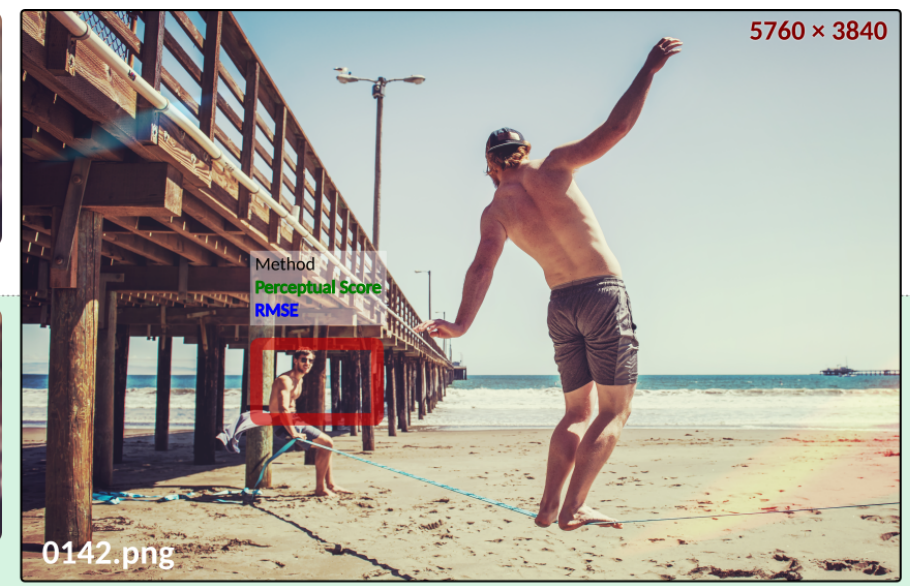

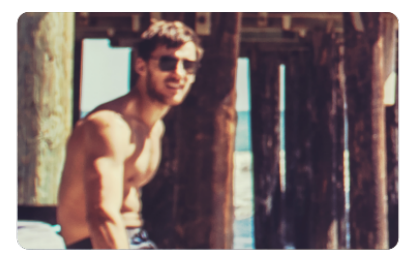

$H R / 6.58 / 0.00$

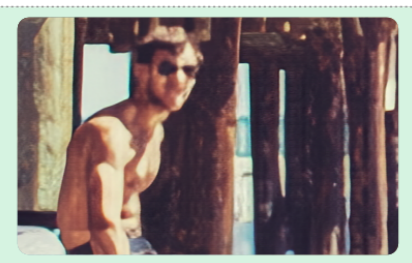

Perceptual / $6.05 / 13.70$

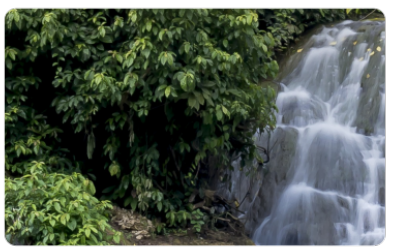

$\mathrm{HR} / 3.18$ / 0.00

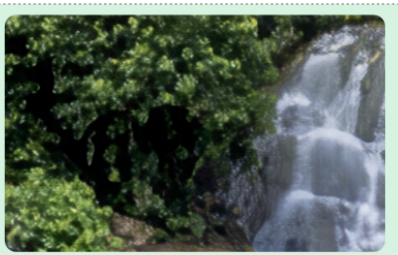

Perceptual / 6.21/ 26.68

Figure 5: Example outputs and performance metrics for images in the DIV8K training set that were used only for validation.

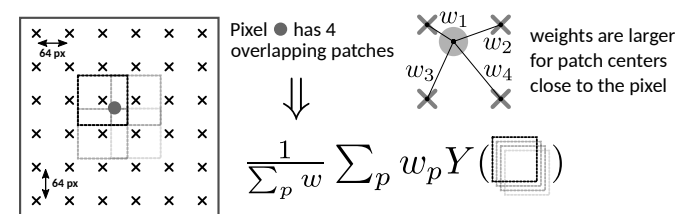

Figure 6: We average overlapping output patches to allow processing arbitrarily large images during inference.

the center of a patch increases (we recommend a Hamming window [6]); and third, we average the results. In our experiments we used overlapping patches separated by 64 and 128 pixels in vertical and horizontal directions for the Perceptual and Fidelity tracks, respectively. The weighted average helps to avoid blocking artifacts.

Another problem arise in the training process because reading several $8 \mathrm{~K}$ images to form a minibatch becomes much slower than running the training step on the minibatch. A popular solution is to save image arrays in a bi- nary format that is much faster to decode (e.g. EDSR [8]). This solution is not practical here since the amount of space required for the whole dataset exceeds reasonable storage resources. We propose a solution by pre-processing the dataset, creating folders for every image in the training set and saving overlapping patches as image files inside each folder, as shown in Figure 7. During training we: first, select images randomly; second, enter the directory and randomly select an image file; third, open the image file and randomly select a patch inside (having similar sizes).

\section{Experiments}

Settings: We run training tasks on Tesla V100 GPUs and testing on Titan X GPUs. We used 100 images from the training set for validation during training. We trained using patch size $667 \times 667$ (Fidelity Track) and $767 \times 767$ (Perceptual Track) at high resolution. We trained our systems using a Quasi-hyperbolic Adam optimizer [10] with constant learning rate $10^{-4}$, and changed to Lookahead [21] 


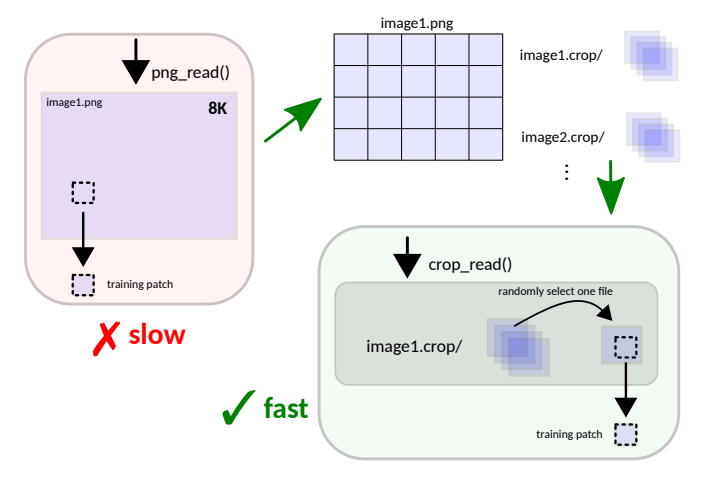

Figure 7: To improve the reading speed in the training process, we replaced images by folders with pre-selected image patches of similar but larger size than training patches.

combined with RAdam [9] in later stages of development. We set the minibatch size to 16 patches. After every epoch we run the validation metrics described above on the center patch of our 100 validation images.

Performance: We report a running time of 47.11 [s] to process a standard $8 \mathrm{~K}$ image $(7689 \times 4320)$ on a Titan X (Maxwell) GPU, without using the overlapping patch approach. It takes $1.42[s]$ in average to process $1000 \times 1000$ images used in the validation stage. For our submissions we use the system in the slowest mode, which uses overlapping patches with small distances between the center of the patches (64 or 128 pixels). By using this approach it takes $4.16[s]$ to process a $1000 \times 1000$ image using $667 \times 667$ overlapping patches with centers separated by 128 pixels (used in the Fidelity track). It takes $2.06[s]$ to process a $1000 \times 1000$ image using $767 \times 767$ overlapping patches with centers separated by 64 pixels (used in the Perceptual track). Overall, in both the Fidelity and Perceptual tracks, it takes approximately 16 minutes to generate one $8 \mathrm{~K}$ output image using the slowest mode. The overlapping patch approach gives marginal improvements in PSNR and also allows the network to run on devices with low memory resources. The settings used for the competition are purposely not practical for applications as they focus exclusively on image quality. Nevertheless, our empirical tests show that by using larger distances between overlapping patches and multiple GPU devices we can output an $8 \mathrm{~K}$ image in less than 10 seconds without significant loss in quality.

Challenge Results: The Fidelity track of the ExtremeSR AIM 2019 challenge had 70 participants, with 9 finalists submitting results for the test stage. Our results obtained the $5^{\text {th }}$ place, with an average PSNR of $26.59 \mathrm{~dB}$ in the full output images of the test set. This is, $0.2 \mathrm{~dB}$ below the top score winning this track.

The Perceptual track of the Extreme-SR AIM 2019 challenge had 52 participants, with 6 finalists submitting results for the test stage. Our results obtained the $1^{\text {st }}$ place in
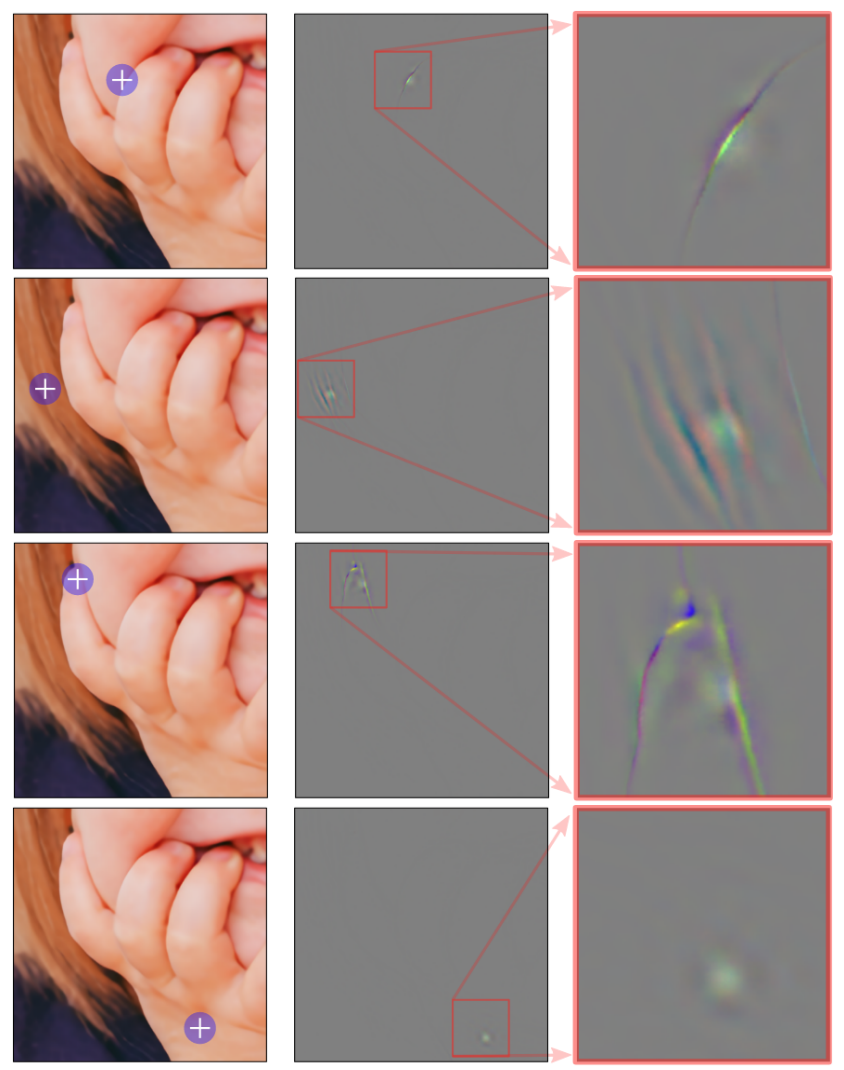

Figure 8: Deep filter visualization (DFV)[16, 14] experiments on our model for the Fidelity track using a patch of size $767 \times 767$. The model shows good knowledge of the geometry and large receptive fields.

terms of a subjective ranking based on mean opinion scores (MOS) to measure perceptual quality. Our results obtained an average PSNR of $25.44 \mathrm{~dB}$ in the full output images of the test set. This is, $0.18 \mathrm{~dB}$ above the $2^{\text {nd }}$ place and 1.35 $\mathrm{dB}$ below the best PSNR value in the Fidelity track.

Figures 5 and 9 show examples of our best results for the Fidelity and Perceptual tracks on images used for validation during our training process. These images show the values of RMSE (measuring fidelity) as well as the Perceptual index proposed in [1] to objectively measure perceptual quality. Overall, the results are consistent with the perception/distortion trade-off in [2]. For the images 142 and 622 we observe that our results for the Perceptual track achieve a Perceptual index better than original images. In image 622 we attribute this result to a blurred background in the original image, that our system shows more focused and with sharper features. In image 622 the results seem to reflect a failure of the Perceptual index as the original image clearly displays a face that our output shows with ambiguous characteristics. This shows that our system does not go as far as to fake people's identities, but it seems to cor- 


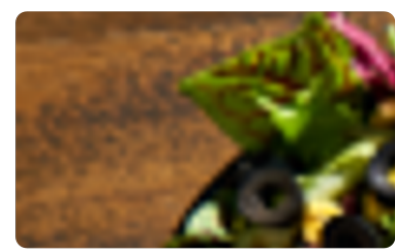

Bicubic /11.09/13.87

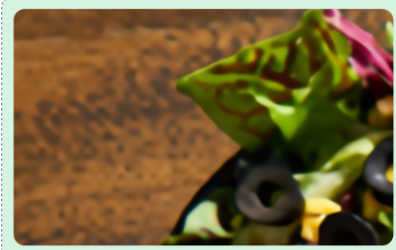

Fidelity / 8.90 / 9.36

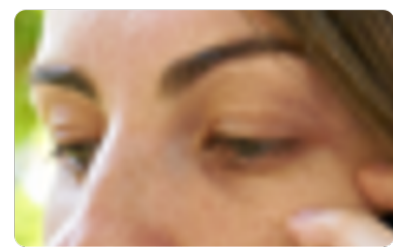

Bicubic /11.26/10.35

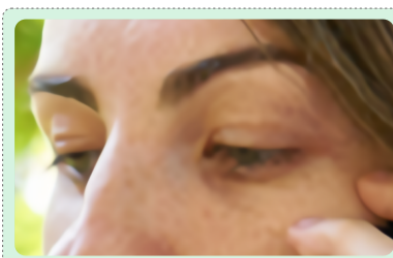

Fidelity / 9.33 / 8.05

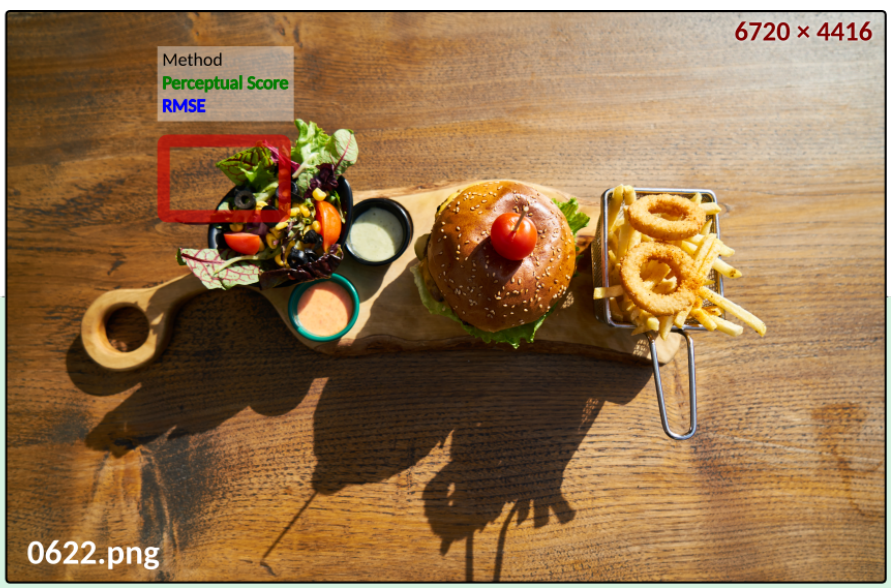

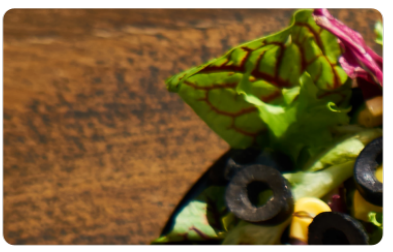

HR / 6.87 / 0.00

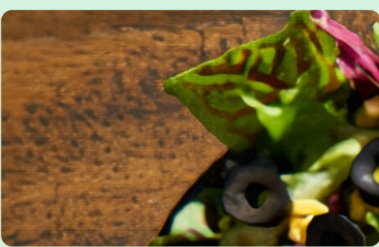

Perceptual / 6.53/11.76

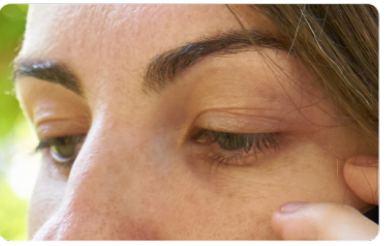

$\mathrm{HR} / 2.88 / 0.00$

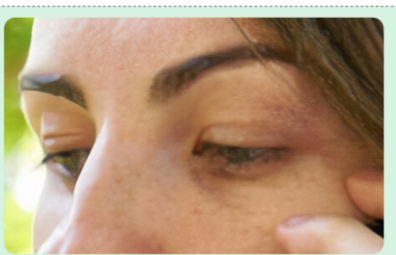

Perceptual /6.23 / 9.11

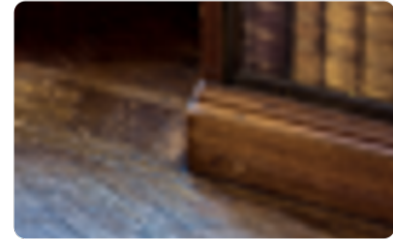

Bicubic /11.19/14.72

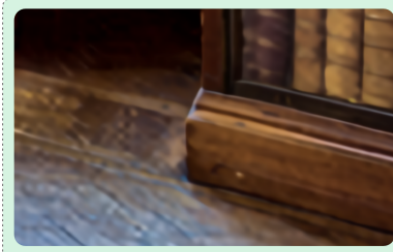

Fidelity / $9.00 / 13.11$

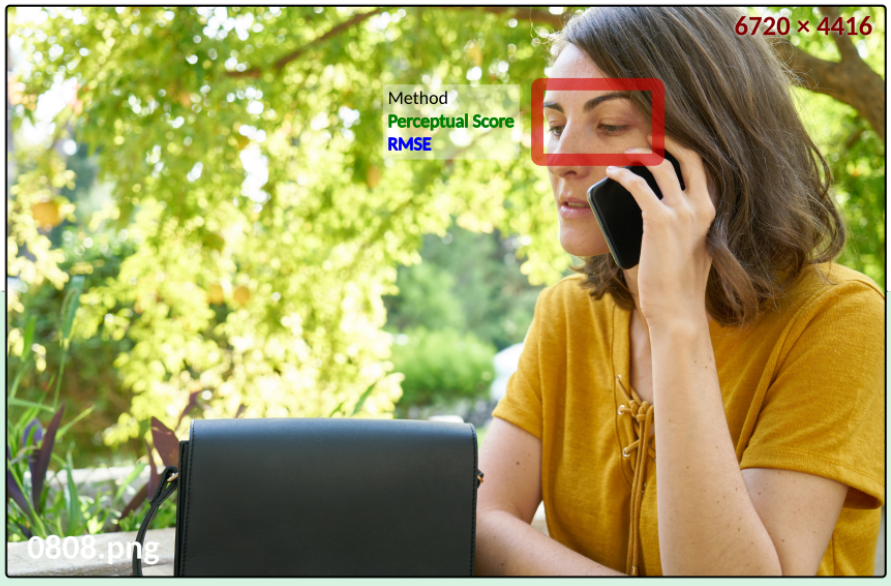

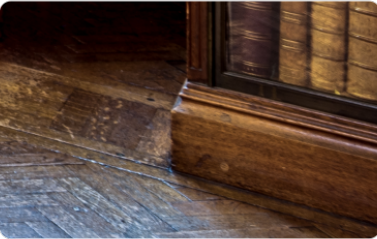

$\mathrm{HR} / 3.67 / 0.00$

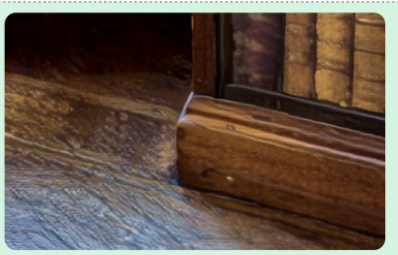

Perceptual /5.27 / 15.88

Figure 9: Example outputs and performance metrics for images in the DIV8K training set that were used only for validation.

rectly identify a person by adding skin and body textures. For images 472, 808 and 1466, the Perceptual indexes are clearly below those of the original image. According to our subjective evaluation, we observe clear differences in the fine level features like: tree leaves and water for image 472; hair, eyebrows and eyelashes in 808; and textbook spines in 1466 . From a far away look these details become less perceptible, indicating that the Perceptual index corre- 

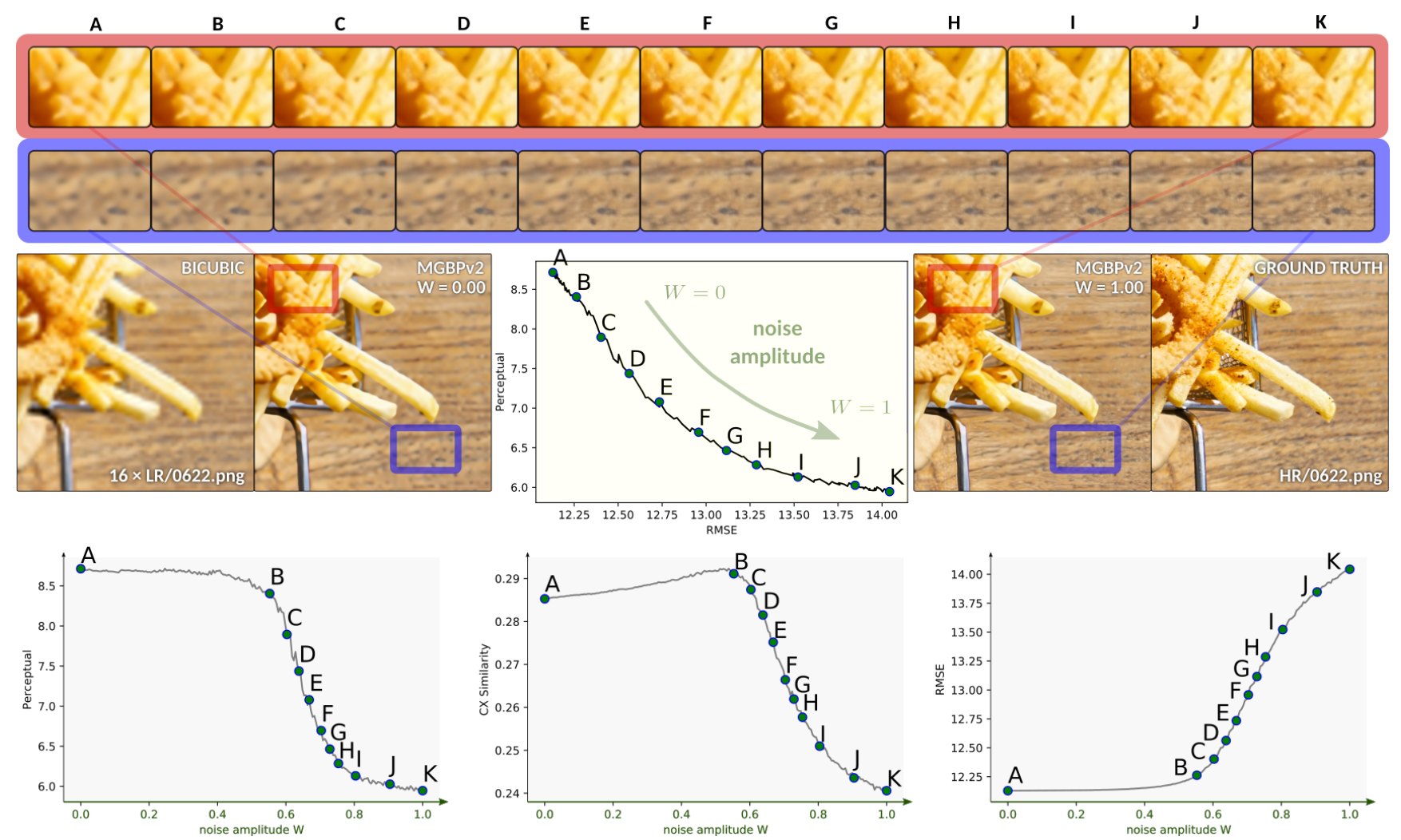

Figure 10: Evolution of perceptual and fidelity metrics when moving the input noise amplitude from $W=0$ to $W=1$.

lates better with a close distance observer. This is probably caused by the resolution of example images used to adjust the Perceptual index, that are much smaller than $8 \mathrm{~K}$.

Figure 10 shows the effect of continuously adding noise in the input of our model for the Perceptual track. We observe that our model has correctly interpreted the target of our loss function, aiming at high fidelity with zero noise amplitude and high perceptual quality for unit noise amplitude. The trajectory in the perception distortion plot is mostly concave, except for the high perception corner. This trajectory is not meant to be optimal as perception/distortion optimality is far from being represented in the loss function. Our loss function only considers the corner cases of zero and unit noise amplitudes. The transition from high fidelity to high perceptual quality is smoother than similar tests performed with MGBP in [15]. The plot for the contextual similarity, as defined in [12], shows the best result in the beginning of the strong transition from high fidelity to high perceptual quality. Before this point it behaves as the perceptual index (improving as noise increases) and after this point it changes to a behavior closer to a fidelity score (worsening as noise increases). Compared to similar experiments performed in [15], the contextual loss here seems to be more focused on preventing low fidelity scores.

Finally, Figure 8 shows interpretability results obtained by using the Deep Filter Visualization (DFV) method from [16]. To perform this complex analysis on a large model such as MGBPv2, with more than 20 million parameters, we use the so-called Linearscope method recently introduced in [14]. For a given pixel in the input image (blue circles on the left side), Figure 8 displays the impulse response for the network model with frozen activations (all ReLU's acting as if the input image did not change). This represents the equivalent to an upscaling filter that adapts to the pixel location. In flat areas (example at the bottom of Figure 8) the upscaling filter looks isotropic and similar to a bicubic upscaler. In other locations, the filter strongly follows edges in hair and fingers, with receptive fields that extend for several hundred pixels. Overall, this confirms that the system has learned the geometry of the content.

\section{Conclusions}

The second generation of Multi-Grid Back-Projection (MGBPv2) networks improves its predecessor by: making better use of the multigrid recursion; simplifying its modules; and redesigning training and inference strategies to improve scalability. MGBPv2 achieves state-of-the-art performance by winning the Perceptual track of the ICCV AIM Extreme-SR Challenge 2019. 


\section{References}

[1] Yochai Blau, Roey Mechrez, Radu Timofte, Tomer Michaeli, and Lihi Zelnik-Manor. 2018 PIRM challenge on perceptual image super-resolution, 2018. 2, 6

[2] Yochai Blau and Tomer Michaeli. The perception-distortion tradeoff. In The IEEE Conference on Computer Vision and Pattern Recognition (CVPR), June 2018. 2, 6

[3] Ran Duan and Pablo Navarrete Michelini. P-47: 10K UltraHigh-Definition display system. In SID Symposium Digest of Technical Papers, volume 47, pages 1294-1296. Wiley Online Library, 2016. 1

[4] The Society for Information Display (SID). 2015 award winners: BOE technology group for its 82-in. 10K display. https://www.sid.org/About/Awards/ PeopleschoiceAwards.aspx, 2015. [Online; accessed 18-Sept-2019]. 1

[5] Muhammad Haris, Greg Shakhnarovich, and Norimichi Ukita. Deep back-projection networks for super-resolution. In IEEE Conference on Computer Vision and Pattern Recognition (CVPR), 2018. 2

[6] Fredric J Harris. On the use of windows for harmonic analysis with the discrete fourier transform. Proceedings of the IEEE, 66(1):51-83, 1978. 5

[7] Alexia Jolicoeur-Martineau. The relativistic discriminator: a key element missing from standard GAN. arXiv preprint arXiv:1807.00734, 2018. 4

[8] Bee Lim, Sanghyun Son, Heewon Kim, Seungjun Nah, and Kyoung Mu Lee. Enhanced deep residual networks for single image super-resolution. In The IEEE Conference on Computer Vision and Pattern Recognition (CVPR) Workshops, July 2017. 5

[9] Liyuan Liu, Haoming Jiang, Pengcheng He, Weizhu Chen, Xiaodong Liu, Jianfeng Gao, and Jiawei Han. On the variance of the adaptive learning rate and beyond. arXiv preprint arXiv:1908.03265, 2019. 6

[10] Jerry Ma and Denis Yarats. Quasi-hyperbolic momentum and adam for deep learning. arXiv preprint arXiv:1810.06801, 2018. 6

[11] Roey Mechrez, Itamar Talmi, Firas Shama, and Lihi ZelnikManor. Learning to maintain natural image statistics, [arxiv](https://arxiv.org/abs/1803.04626). arXiv preprint arXiv:1803.04626, 2018. 4

[12] Roey Mechrez, Itamar Talmi, and Lihi Zelnik-Manor. The contextual loss for image transformation with non-aligned data. arXiv preprint arXiv:1803.02077, 2018. 4, 8

[13] A. Mittal, R. Soundararajan, and A. C. Bovik. Making a "Completely Blind" Image Quality Analyzer. IEEE Signal Processing Letters, 20:209-212, Mar. 2013. 4

[14] Pablo Navarrete Michelini, Hanwen Liu, Yunhua Lu, and Xingqun Jiang. A tour of convolutional networks guided by linear interpreters. In The IEEE International Conference on Computer Vision (ICCV). IEEE, Oct 2019. 6, 8

[15] Pablo Navarrete Michelini, Hanwen Liu, and Dan Zhu. Multi-scale recursive and perception-distortion controllable image super-resolution. In The European Conference on Computer Vision Workshops (ECCVW), September 2018. 2, $3,4,8$
[16] Pablo Navarrete Michelini, Hanwen Liu, and Dan Zhu. Multigrid backprojection super-resolution and deep filter visualization. In Proceedings of the Thirty-Third AAAI Conference on Artificial Intelligence (AAAI 2019). AAAI, 2019. $2,6,8$

[17] Gu Shuhang, Martin Danelljan, Radu Timofte, et al. AIM 2019 challenge on image extreme super-resolution: Methods and results. In The IEEE International Conference on Computer Vision (ICCV) Workshops, October 2019. 1

[18] M. Sugawara, Choi S-Y, and D. Wood. Ultra-high-definition television (Rec. ITU-R BT.2020): A generational leap in the evolution of television. Signal Processing Magazine, IEEE, 31(3):170-174, May 2014. 1

[19] Ulrich Trottenberg, Cornelius W Oosterlee, and Anton Schuller. Multigrid. Academic Press, Inc., Orlando, FL, USA, 2001. 2

[20] Xintao Wang, Ke Yu, Shixiang Wu, Jinjin Gu, Yihao Liu, Chao Dong, Yu Qiao, and Chen Change Loy. ESRGAN: Enhanced super-resolution generative adversarial networks. In Proceedings of the European Conference on Computer Vision (ECCV), pages 0-0, 2018. 4

[21] Michael R Zhang, James Lucas, Geoffrey Hinton, and Jimmy Ba. Lookahead optimizer: $\mathrm{k}$ steps forward, 1 step back. arXiv preprint arXiv:1907.08610, 2019. 6 